Voix et Images

\title{
Les hauts et les bas d'une grande amitié littéraire : Louis \\ Dantin-Alfred DesRochers (1928-1939)
}

\section{Annette Hayward}

Volume 16, numéro 1 (46), automne 1990

Les correspondants littéraires d'Alfred DesRochers

URI : https://id.erudit.org/iderudit/200871ar

DOI : https://doi.org/10.7202/200871ar

Aller au sommaire du numéro

Éditeur(s)

Université du Québec à Montréal

ISSN

0318-9201 (imprimé)

1705-933X (numérique)

Découvrir la revue

Citer cet article

Hayward, A. (1990). Les hauts et les bas d'une grande amitié littéraire : Louis Dantin-Alfred DesRochers (1928-1939). Voix et Images, 16(1), 26-43.

https://doi.org/10.7202/200871ar d'utilisation que vous pouvez consulter en ligne. 


\section{Les hauts et les bas d'une grande amitié littéraire: Louis Dantin- Alfred DesRochers (1928-1939)}

\section{par Annette Hayward, université Queen's}

La correspondance entre Louis Dantin et Alfred DesRochers tire son origine de l'enthousiasme avec lequel ce vieux critique "en exil" accueillit le recueil l'Offrande aux vierges folles que DesRochers lui avait envoyé le 3 décembre 1928.

À l'époque, Alfred DesRochers souffrait beaucoup de l'incompréhension et de l'indifférence que ce premier recueil rencontrait auprès du public. Le 29 juillet 1929, il avoue à Dantin:

J'en ai envoyé un exemplaire à tous les journaux français de

la province à peu près [...] Tout ce qu'on a convenu d'appeler

la critique officielle, sauf Albert Pelletier, dans la Revue mo-

derne, et vous-même, n'en a soufflé mot.

On peut donc facilement imaginer le plaisir du jeune DesRochers lorsqu'il reçut la première lettre de Louis Dantin, datée du 31 décembre 1928, exprimant une grande admiration pour ces vers pleins d'émotion qui se caractérisent non seulement par la beauté plastique, mais aussi par $d u$ sens, du mysticisme et [...] une sorte de profondeur navrante. Le critique déclare même son intention d'en faire une étude pour le Canada, dès qu'il se sera dépêtré des monceaux d'œuvres qu'il lui reste à apprécier. Il signale cependant quelques fautes [...] noyées dans une masse de vers superbes et en cite en post-scriptum un exemple particulièrement frappant.

Comment rester insensible à de tels éloges de la part d'un aîné qui publie dans la Revue moderne et le Canada, qui avait fait la célèbre préface à Émile Nelligan et son œuvre (1904) et qui vient tout juste de faire paraitre un volume sur les Poètes de l'Amérique française (1928)? Pressentant chez lui un mentor prêt à le conseiller, DesRochers réplique immédiatement en envoyant deux nouvelles poésies, dont "Hymne au Vent du Nord". Dantin reconnait tout de suite dans ce long poème une œuvre lyrique de premier ordre et le renvoie avec maintes annotations. L'importance de ces remarques pour DesRochers se mesure au fait que, lorsque l'on compare l'édition finale du poème au manuscrit dactylographié annoté par Dantin, on 
voit qu'elles sont à l'origine de la plupart des changements importants apportés au texte. DesRochers ne suit pas aveuglément les conseils du maître, cependant; il écarte, entre autres, certaines réticences de Dantin à l'égard du manque de réalisme ou de nuance de certains vers.

Ce sera ensuite au tour de Dantin de soumettre à DesRochers, avec qui il adopte déjà un ton de connivence, un de ses propres poèmes, une fantaisie rimée à lui, en argot. Il s'agit de la future Chanson javanaise, trop osée, selon l'auteur, pour qu'on puisse songer à la faire publier au Canada.

Ainsi débute une correspondance qui s'échelonnera sur une période de onze ans, de décembre 1928 à décembre 1939, mais avec quatre ans de quasi-silence entre novembre 1934 et janvier $1939^{1}$. Ces lettres nous plongent non seulement dans le monde littéraire des années trente au Québec, mais aussi dans la vie intime de deux hommes exceptionnels qui y ont joué un rôle essentiel. Elles nous donnent un aperçu de leurs préférences littéraires et de leurs jugements sur d'autres écrivains québécois. Elles nous révèlent en outre l'existence de projets restés inachevés, ainsi que de motivations parfois insoupçonnées qui auraient présidé à certains écrits. On y trouve des détails sur le monde de l'édition d'alors, sur les effets de la Crise et surtout sur les conséquences du moralisme de l'institution littéraire de l'époque. Elles donnent non seulement une idée de l'influence exercée par Dantin sur DesRochers mais aussi, ce qui est moins connu, du rôle primordial joué par ce dernier dans la carrière littéraire de son aîné.

\section{Deux âmes sœurs}

$\grave{A}$ vrai dire, la correspondance entre DesRochers et Dantin se lit un peu comme une histoire d'amour, un coup de foudre entre deux êtres qui se découvrent des âmes sœurs. Avec le temps, cependant, des différences fondamentales se manifestent qui, malgré la bonne volonté des deux écrivains, finissent par les éloigner l'un de l'autre...

Ainsi assiste-t-on à une première période de révélations progressives, de confidences d'abord timides, puis de plus en plus franches.

1 Le fonds DesRochers, sur lequel porte surtout notre analyse, compte 122 lettres (en plus d'une carte de souhaits et d'un télégramme) de Louis Dantin, joliment écrites à la main et presque exemptes de ratures ou d'erreurs de français. On y trouve également onze lettres de DesRochers: deux sur lesquelles Dantin avait écrit sa propre reponse et neuf que DesRochers avait cru assez importantes pour en conserver des copies. Celles-ci, comme les 104 lettres-de DesRochers à Dantin (plus deux cartes) conservées dans le fonds Nadeau, sont souvent tapées à la machine (une machine sans accents, au début) et trahissent parfois une vie mouvementée, aux interruptions fréquentes. 
Ils se comprennent certes, ce jeune poète fougueux de 27 ans et ce vieux critique-écrivain exilé de 64 ans $^{2}$, mais, surtout, chacun comprend la difficulté pour quelqu'un aux idées un peu modernes de se faire publier au Québec, d'y trouver un public et d'y faire reconnaître son talent, voire son génie.

Leurs premiers échanges seront d'ordre littéraire, là où, au moins au début, l'accord entre les deux semble presque parfait. En effet, lorsque Dantin fait le compte rendu de l'Offrande aux vierges folles, en juillet 1929, il déclare:

Voici un jeune poète qui se fait de la poésie la conception que j'en ai moi-même. Il la voit, il la sent comme une exaltation intime, comme la surprise de l'âme en présence du monde, comme un effluve de pensées hautes et d'émotions intenses, comme un élan vers la Beauté, comme un soupir exhalé vers le rêve; mais il ne commet pas l'erreur de la placer toute en cela. Il sait qu'à ce stade primitif elle n'est qu'en puissance et en germe; qu'il faut, pour nous atteindre, qu'elle s'exprime par des signes et des symboles; que c'est le langage seul qui, en fait, la réalise. Il comprend [...] qu'en suscitant le mot logique, expressif, évocateur, c'est la pensée même qu'il projette, qu'il précise, $q u$ 'il resserre, $q u$ 'il fait resplendir. ${ }^{3}$

De la même façon, DesRochers se montrera fort enthousiaste devant l'idylle-satire trop osée de son confrère, "Le Canadien errant" (future Chanson javanaise), et quand Dantin se propose de la lui léguer, satisfait d'avoir un lecteur au moins qui s'y sera un peu reconnu (6 avril 1929), le poète de Sherbrooke s'engage à la publier pour l'édification des lettres, sur papier de luxe, aussitôt que ses finances le permettront. C'est ce qu'il fera, avant la fin de l'année, malgré les craintes de son ami (qui refuse de signer autrement que par ses initiales), de façon à ce que Dantin puisse l'offrir en étrennes pour le Nouvel An.

Entre-temps, DesRochers aura soumis à Dantin une bonne partie des poèmes qui constitueront son deuxième recueil, alors intitulé Ma province aux noms exotiques. Les réactions du critique sont mitigées, allant d'une approbation absolue de l'«Hymne au Vent du Nord", de "Soir d'été à Saint-Denis de Brompton " et de la "Prière au Bon Dieu des gens frustres de chez nous ", ou de quelques réserves au sujet des sonnets terroiristes du "Cycle des bois et des champs" (Dantin trouve la forme trop rigide pour le sujet traité), jusqu'à une

2 Toutes les sources que nous avons consultées, à l'exception du Dictionnaire des œuvres littéraires du Québec, tome II, 1980, p. 893, qui indique 1869, donnent 1865 comme date de naissance de Louis Dantin.

3 Louis Dantin, Poètes de l'Amérique française. Études critiques (II' série), Montréal, Éditions Albert Lévesque, 1934, p. 98-99. 
réaction carrément négative à l'égard du poème "Ma province aux noms exotiques ». Dantin se rebiffe devant l'emploi de noms anglais et amérindiens comme noms exotiques; d'abord, dit-il, parce que les noms indiens n'en sont point, selon l'acception stricte du terme, et ensuite parce que les noms anglais ne font qu'évoquer la faiblesse d'un peuple vaincu. DesRochers répond en avouant qu'il avait tout simplement considéré le mot exotique comme synonyme d'étranger, en estimant que, pour tout francophone, les noms anglais et amérindiens étaient des noms étrangers. Il se révèle ainsi moins puriste et savant que Dantin, mais peut-être aussi moins complexé vis-à-vis des "Anglais". L'auteur précise également que la province en question n'est pas le Québec, comme Dantin semble le croire, mais les cantons de l'Est, sa province à lui. Suit un passage essentiel qui explique en partie l'ambition qui motivait DesRochers à cette période de sa vie:

Vous avez vécu au Canada, vous ne devez jamais avoir entendu parler des Cantons de l'Est et de Sherbrooke, ailleurs que dans les réclames de chemins de fer. Il est stipulé tacitement que dans la province de Québec il existe deux villes. - Dans l'une vivent les nombrils d'univers, c'est Montréal, dans l'autre, les imbéciles, c'est Québec. Le reste, c'est la campagne.

Avec l'orgueil dont je brûle, mais dont je ris tout haut en public, je sens que je puis bâtir une œuvre dans laquelle il y aura plus de souffle et plus de véritables vers que n'en peuvent écrire les virtuoses de l'adjectif de Montréal ou les pontifes de l'heure des vaches de [Québec]. J'ai l'ambition de mettre mes Cantons sur la carte, comme nous disons à l'anglaise. (11 avril 1929)

La conception du poème "Ma province aux noms exotiques montre en effet que DesRochers avait bien compris une des ironies de la querelle entre les régionalistes et les "exotiques" qui sévissait au Québec depuis plus de dix ans. Les régionalistes, en voulant qu'on décrive de façon idéaliste les particularités qui différencient le Québec du reste du monde, rejoignaient en fait les visées de l'exotisme, genre où l'on évoque surtout l'aspect unique, "exotique", d'un pays étranger. En produisant une œuvre où le titre évoquait en même temps le régionalisme ("Ma province [...]") et l'exotisme, mais où la plupart des poèmes représentaient ses compatriotes et ses ancêtres de façon fort réaliste, DesRochers se distinguait des deux camps et particulièrement du régionalisme ancien style.

Devant les remarques de Dantin, DesRochers abandonne le poème "Ma province [...]" et, par corollaire, le titre prévu pour son nouveau recueil. L'idée du nouveau titre, A l'ombre de l'Orford, viendra sans doute d'un autre projet qu'il mijotait à la même époque, "La prosopopée de l'Orford", où il se proposait de faire parler le mont 
Orford comme une sorte de dieu qui aurait assisté à toutes les splendeurs et les obscurités de mon petit pays.

On peut douter, cependant, que DesRochers ait accepté la critique que fait Dantin de "Ma province [...]" avec autant de facilité que sa lettre du 11 avril 1929 voudrait le laisser entendre 4 . Cette semaine-là justement, il avait - comme par hasard - relu dans la Revue moderne d'antan les poèmes et les contes qu'y avait publiés Dantin. Il en profitera pour encourager le critique à les publier en volume, ne serait-ce que pour montrer que dans vos œuvres, vous avez autant et mieux que d'autres le génie "créateur" et que quand vous recommandez qu'on mette de la forme à ce qu'on écrit, vous savez ce dont vous parlez (11 avril 1929). N'est-ce pas plutôt DesRochers luimême qui voulait s'assurer que Dantin savait ce dont il parlait avant d'accepter ses critiques? Si tel est le cas; ces anciens contes, joints à Chanson javanaise, semblent l'avoir rassuré et les relations entre les deux hommes n'en seront que raffermies. Puis, leurs rapports deviendront encore plus intimes après la rédaction de l'article de Dantin sur l'Offrande aux vierges folles, alors que DesRochers se met à raconter ses rêves et ses multiples projets et que Dantin commence à envoyer à son nouvel ami ses écrits anciens èt récents, osés ou orthodoxes, certain de trouver chez lui une oreille sympathique et approbatrice.

Les deux s'excusent d'aillleurs d'une telle intimité avec quelqu'un qu'ils connaissent si peu. Ils avouent céder à une attirance irrésistible, irrationnelle ${ }^{5}$, ce qui laisse deviner le besoin que cette nouvelle fraternité comblait dans leur vie.

D'e 1929 à 1931, Alfred DesRochers passe par une période de productivité intense. Il veut tout faire, semble-t-il. En plus de son travail accaparant comme agent, puis directeur (1930) du service de la publicité à la Tribune, il écrit de la poésie (deux recueils en deux ans) et des critiques (articles dans la Tribune et le volume Paragraphes), il entretient une correspondance volumineuse et des amitiés fort variées, il organise des rencontres littéraires à Sherbrooke, participe à d'autres à Montréal et à Québec, et envisage des projets de toutes sortes. L'institution littéraire québécoise est au centre de presque toutes ses préoccupations. Il sent qu'il peut y apporter une contribution importante et il voudrait associer Dantin,

4 Au sujet du sens du terme "exotique", par exemple, DesRochers affirme: [...] je n'ai jamais consulté mon dictionnaire - je ne l'ai pas encore fait, m'en tenant à vos indications [...] (11 avril 1929).

5 Je ne sais pourquoi il me revient toujours de vous faire une sorte d'exécuteur testamentaire de ma vie littéraire manquée et vieillissante. (Dantin, 22 juillet 1929) Je ne sais si je vous intéresse [...] mais [...] maintenant que je reçois un peu de sympathie de quelqu'un en qui jai foi, je ne puis résister à la tentation de lui tout devoiler de mes ambitions. (DesRochers, 29 juillet 1929) Nous soulignons. 
injustement exilé, à sa tentative. Lui-même se sent isolé des grands centres de Québec et de Montréal et s'évertue à créer autour de lui, dans les cantons de l'Est, un milieu littéraire et intellectuel stimulant. Tout lui semble possible à cetté époque.

Dans le feu de sa nouvelle amitié pour Dantin, DesRochers s'efforce tout d'abord d'aider à la publication des écrits de son aîné: il fait imprimer Chanson javanaise à ses propres frais, prend des dispositions pour l'impression de Chanson citadine, encourage Dantin à préparer un recueil de contes (la Vie en rêve) et de poèmes (le Coffret de Crusoé) et ensuite en assure l'édition, avec le premier volume des Gloses critiques, auprès d'Albert Lévesque. Il essaie aussi de le convaincre de briguer le poste de directeur littéraire à une chaîne de journaux (la Tribune, le Soleil, la Patrie) dont son patron, Florian Fortin, devait devenir, dit-il, le gérant-général. Sa lettre du 29 juillet 1929, en particulier, qui suit la lecture de l'article de Dantin sur l'Offrande, constitue un exemple saisissant de la force de l'amitié offerte par DesRochers à son aîné.

Louis Dantin se révèle lui aussi très actif et fort productif. Il voudrait publier ses œuvres de création ${ }^{6}$, mais il se méfie du moralisme excessif des instances de consécration québécoises. Il se hâte donc, dès qu'il sent la force de l'amitié que lui voue DesRochers, de léguer ses œuvres "posthumes" au poète de Sherbrooke pour que celui-ci les publie plus tard, quand le milieu s'y prêtera mieux ${ }^{7}$. DesRochers répond, comme on sait, qu'il veut les publier tout de suite, èt on constate que Dantin se laisse facilement convaincre. Pour Chanson javanaise, par exemple, il exprime quelques craintes, mais ajoute tout de suite ses spécifications quant au choix du papier, des caractères, etc., et il accepte de signer le texte de ses initiales. L'année suivante, c'est lui-même qui propose à DesRochers de faire imprimer à compte d'auteur Chanson citadine.

Malgré toute cette activité, Louis Dantin et Alfred DesRochers se plaignent souvent de leur travail éreintant qui laisse peu de temps ou d'énergie à consacrer à la littérature. En effet, leur situation n'est pas facile, surtout quand les effets de la Crise commencent à se faire sentir et que DesRochers, en particulier, peine à faire vivre une famille de six enfants.

6 Le 21 juin 1935, Dantin dit regretter d'avoir consacré tant de temps à la critique plutôt qu'à ses propres créations.

7 Peut-être, vous qui êtes jeune, verrez-vous le jour où des peintures sincères et avec des dessous sérieux, comme je crois qu'est celle-ci, n'effaroucheront plus la conscience publique. (19 avril 1929) Les fréquentes sorties de Dantin à ce sujet, qui reviennent dans au moins vingt-quatre de ses lettres à DesRochers, constituent un véritable réquisitoire contre l'institution littéraire d'alors, trop contrôlée par le clergé. 
N'empêche qu'à force d'entendre Dantin parler de son inertie mentale, de ses dépressions et de voir revenir comme un refrain des propos tels que jai un travail de chaque jour brutal et absorbant; je n'ai pour autre chose que des minutes, avec des nerfs épuisés et une tête ahurie (24 mars 1929), et de remarquer qu'il continue quand même à produire régulièrement des textes ainsi qu'une correspondance volumineuse, on finit par se demander si ces remarques, tout en étant sincères, ne faisaient pas un peu partie du "personnage Louis Dantin ». Car il existe un personnage Louis Dantin, dont lui-même est conscient $^{8}$, une image plutôt terne dont il protège soigneusement la respectabilité (30 avril 1939). Cet être fataliste, résigné, un peu désabusé, qui parle souvent de sa vie manquée, c'est en partie luimême, bien sûr. Mais il y a un autre côté, tout aussi vrai: il y a l'homme resté idéaliste et entier, qui voue un véritable culte à la littérature, au Beau et au Vrai, qui se rebiffe devant un catholicisme étouffant et foncièrement capitaliste ${ }^{9}$, et qui a l'audace non seulement de se mêler des choses littéraires du Québec, mais aussi de réagir assez fortement (tout en adoptant un ton raisonnable et pacifique) devant ce qu'il considère comme la bêtise, l'erreur ou la lâcheté.

Loin de nous, cependant, la prétention de régler la question de l'identité problématique de ce Québécois surdoué dont la vie, à en juger par ces lettres, se lit comme un véritable roman. Et encore, il ne souffle mot du renoncement au pouvoir qui dut accompagner sa crise spirituelle et amoureuse alors qu'il était devenu, à 27 ans, supérieur de la maison des pères du Saint-Sacrement à Paris.

Ce qui est indiscutable, c'est que Louis Dantin constitue un interlocuteur extrêmement cultivé, qui apporte à cette correspondance une grande expérience de la vie et une longue réflexion sur la littérature. Quant au jeune DesRochers, qui n'a jamais fini un cours classique entrepris sur le tard, il y apporte surtout l'originalité de sa pensée et la force de ses convictions. Lui aussi a beaucoup réfléchi à la littérature, comme en témoignent ses remarques sur les possibilités énormes qu'aurait l'œuvre de "raté» (genre Jehan Rictus); sur le fond et la forme qui sont tout un (5 avril 1929); sur l'économie des moyens comme l'unique méthode de créer chez le lecteur le sentiment ou l'état d'esprit qu'on désire; ou sur l'importance de l'apport du

8 Au moment où les deux amis échangent des photographies, Dantin envoie d'abord une photo récente où il a l'air dur et desséché (peut-être par l'habitude, comme il le dit, de cacher son être réel aux autres [8 décembre 1929]), puis fait faire pour son nouvel ami la photogravure d'une photo ancienne qui le montre tel qu'il était à 38 ans, et qu'il affirme être plus lui-même que l'être passé que je suis devenu (1 $1^{\text {er }}$ juin 1930).

9 Il décrit l'Église catholique comme la plus grande force d'oppression qui pèse sur les droits de l'esprit, sur les instincts de la nature, sur l'évolution normale de l'humanité (29 décembre 1929). 
quatuor des jeunes filles - Jovette, Simone, Alice et Éva ${ }^{10}$ à la poésie canadienne (3 mai 1929). Ailleurs, il affirme que la poésie est une œuvre d'art plastique, chose parfaitement inutile, et que le théâtre est peut-être le seul moyen de faire servir le vers à de grandes idées (29 juillet 1929). Voilà pourquoi, dit-il, il travaille à ce volume de vers terroiristes (À l'ombre de l'Orford): il s'agirait d'un pur exercice, pour maîtriser le vers et montrer qu'on peut en faire sur tout sujet ${ }^{11}$. Plus tard, il espère écrire un drame sur "Chiniquy " qui lui permettrait d'exprimer à travers un personnage des idées qu'il ne peut avancer (par crainte de censure? ou de scandale?) en son propre nom.

\section{Premiers désaccords}

Ce projet sur Chiniquy, confié à Dantin dans un élan de confiance le 29 juillet 1929 , servira non pas à rapprocher les deux hommes mais, ironiquement, à faire ressortir les différences philosophiques et métaphysiques fondamentales qui les séparent. Dantin s'identifie de façon évidente à ce Québécois énigmatique qui fut un des grands orateurs religieux du XIX ${ }^{\text {e }}$ siècle avant d'apostasier publiquement et de se mettre à convertir ses compatriotes au protestantisme, alors que DesRochers, dont l'attitude est pourtant un peu ambiguë, le voit essentiellemet comme un hypocrite et un lâche. Cette discussion provoque des révélations sur la position de Dantin par rapport à l'Église que DesRochers, en tant que bon catholique, a du mal à accepter.

Par la suite, Dantin préfere éviter le sujet de la religion, n'ayant aucune envie, dit-il, de convertir son jeune ami. Mais lorsque DesRochers refuse son offre des Confessions de Jean-Jacques Rousseau sous prétexte que cette œuvre est à l'Index, son ami ne peut retenir une véritable diatribe contre cette Église qui interdit à ses fidèles plus de la moitié des chefs-d'œuvre de la littérature française, qui réduit en esclavage l'intelligence humaine et lui défend d'aimer la vérité, la beauté pour elles-mêmes (29 décembre 1929). Puis il s'en "excuse et termine avec l'assurance qu'il respecte toute personne logique avec sa propre conscience. Cette tirade aura comme conséquence que DesRochers acceptera de lire les Confessions. Mais quand ce dernier essaie de prendre la défense de l'Index, Dantin refuse de rouvrir un débat qui ne peut être que futile, dit-il, entre deux esprits séparés par les antipodes (9 janvier 1930).

10 Il s'agit de Jovette-Alice Bernier, Simone Routier, Alice Lemieux et Éva Senécal.

11 Dans la même lettre, DesRochers se contredit au moins partiellement en disant qu'il tente aussi dans l'Orford de battre en brêche, sans en avoir l'air, l'idéalisme et l'irréel dont s'est entourée notre production régionale. Il admet même que cela le touche de près, car il est lui-même issu de ces rudes travailleurs. Tout cela est assez loin donc du pur exercice gratuit et se situe plutôt dans la lignée de, la querelle entre les régionalistes et les «exotiques ». DesRochers semble vouloir libérer le régionalisme des entraves idéologiques qui l'empêchent de produire des œuvres valables. 
Le cas des Confessions n'est pas l'unique occasion où DesRochers se laisse convaincre par les arguments du maître. Sur la question du socialisme soulevée par la Crise, par exemple, la conviction assez simpliste ${ }^{12}$ de Dantin, un socialiste ou même un communiste de longue date, finit par avoir gain de cause sur les hésitations de DesRochers. Celui-ci, qui projetait un livre sur le Rôle économique du journal dans la vie moderne, dit se méfier du socialisme (16 novembre 1931) et se lance dans de grandes lectures en économie politique. Dantin, qui semble bien connaître la matière, répète avec insistance que tout cela est du temps perdu et que la confusion qui règne dans ce domaine se dissipe devant la simplicité, la cohérence et la logique du socialisme, à savoir: assurer un maximum de bien-être au plus grand nombre et mettre tout le monde à l'abri du dénuement, par la coordination et la coopération (27 mai 1932). Le 18 avril 1933, DesRochers mettra fin à ses tergiversations et annoncera même son intention de fonder une feuille socialiste au Québec, entreprise risquée que son vieil ami, malgré son bonheur devant une telle conversion, décourage fortement (28 avril 1933).

La confrontation la plus violente entre les deux amis aura cependant pour thème le canadianisme intégral, une école de pensée qui annonce étrangement certaines théories sur la décolonisation qui auront cours au Québec durant les années soixante et soixante-dix. Albert Pelletier, un des principaux porte-parole de cette théorie, selon DesRochers ${ }^{13}$, prône par exemple une langue canadienne et insiste sur le fait que le Canadien d'aujourd'hui a autant d'affinités avec la culture américaine qu'avec celle de la France. De telles affirmations provoquent une réaction déchaînée chez Dantin, le 24 mars 1931, auquel DesRochers se résoudra enfin à répondre, le 26 juin suivant. Lorsqu'il explique de son mieux, avec une grande franchise, sa sympathie pour de telles théories, Dantin excédé riposte sur la même feuille.

Cette explication (26 juin 1931) est importante pour comprendre, entre autres, À l'ombre de l'Orford. Le poète se définit d'abord par rapport à la France, en affirmant que nous devons nous assimiler l'essence de sa culture, mais toutes nos applications doivent être canadiennes. Il ne va pas jusqu'à prôner une langue canadienne ${ }^{14}$ et

12 Rêves poétiques ou rêves socialistes, c'est, après tout, le même idéal, qui vous fait chercher quelque chose au-dessus de la matiere brute et des institutions brutales. (28 avril 1933)

13 Alfred DesRochers, Paragraphes (Interviews littéraires), Montréal, Librairie d'action canadienne-française, 1931, p. 178. Voir aussi l'article de Richard Giguère, supra.

14 DesRochers dit néanmoins que, idéalèment, il aimerait voir au Québec une évolution en silence, d'où sortiraient un Rabelais, un Montaigne, un Ronsard. Faudrait-il ajouter, par corollaire, et une langue canadienne? 
affirme que, puisque nous n'avons pas de langue à nous, il faut exploiter notre substance. C'est ce qu'il croit avoir fait dans son œuvre:

[...] il me semble que même dans mes vers les moins terroirisants, je suis plus canadien que toute l'école de Québec; car il me semble que les inquiétudes dont je parle, les aspirations que j'énonce, sont essentiellement du tempérament canadien.

DesRochers se laisse peut-être emporter un peu quand il fait une sortie contre les Français centralisateurs et chauvins ou lorsqu'il affirme que, à égalité de talent, une œuvre américaine ou anglaise a plus de résonances profondes pour un Canadien français qu'une œuvre française. Il faut cependant reconnaitre que l'essentiel de sa théorie vise à libérer le régionalisme québécois, si porté vers le moralisme et l'idéalisme, des entraves idéologiques qui l'empêchaient d'évoluer vers le réalisme.

Dantin, totalement réfractaire à ces propos, y voit une théorie mesquine qui veut entraver la liberté de l'artiste (Défense au génie de sortir des frontières du Québec!) et il nie que l'œuvre de DesRochers comporte quoi que ce soit d'essentiellement ou d'exclusivement canadien. 'Quant à l'idée qu'un Canadien français puisse préférer une œuvre américaine ou anglaise à une œuvre française (adjectif qu'il interprète comme synonyme de canadienne-française, ce qui n'était sûrement pas l'intention de DesRochers), il y voit une confession de défaite, une acceptation bénévole de la part des vaincus, la fin de la littérature canadienne-française et de la "race».

DesRochers revient à la charge, le 29 juin 1931, dans un effort pour se faire comprendre:

La discussion que nous avons entreprise en est une pratiquement sans issue et je dois reconnaître que vous êtes meilleur logicien que moi. Vos arguments me désarment... mais le pragmatique demeure inconvaincu. C'est le propre de la vie d'échapper à toute logique [...].

Il s'explique de nouveau, affirmant entre autres que ces moyens préconisés pour créer un esprit (et, corollairement, une littérature) national sont des moyens passagers: plus tard [...] nous pourrons faire ce que nous voudrons. Quant à l'essence canadienne de son œuvre à lui:

Et vous avez raison quand vous dites que mes sonnets bûcherons ont la plus mince couche de canadianisme que connaisse notre poésie [en de pareils sujets], justement parce que ce sont des œuvres "exotiques", faites par gageure, d'après un procédé; tandis que mon "Hymne au Vent du Nord", même mon "Ode au crapaud" me semblent traduire quelque chose 
de foncièrement canadien, en ce qu'elles exaltent l'underdog, ceux qu'il est de bon goût de mépriser ou de calomnier. (29 juin 1931)

Le 7 juillet suivant, lorsque Dantin écrit pour accepter l'invitation de DesRochers (datée du 3 juillet) de venir, toutes dépenses payées, assister à une réunion d'écrivains qui aura lieu à Sherbrooke le 18, il refuse de reprendre l'amicale discussion. Il dit se consoler par le fait que DesRochers et tous les jeunes canadianisants écrivent en réalité sur tous les sujets, et dans le français le plus français que vous puissiez atteindre. C'est tout ce que la littérature canadienne vous demande; et elle vous pardonne, moyennant cela, toutes les chimères possibles...

Malgré cette tentative de simuler la tolérance et l'indifférence, Dantin ne peut résister à la tentation de revenir sur ce sujet et c'est ainsi qu'il prononcera à Sherbrooke, le 18 juillet 1931, sa conférence sur "La langue française, notre instrument d'expression littéraire " 15 . Il y prône l'emploi de la langue française comme condition sine qua non de la survivance de la littérature et de la nation canadiennesfrançaises. Dans sa lettre récapitulative du 2 août 1931 , où il doute de pouvoir (vouloir?) assister de nouveau à une réunion à Sherbrooke, il se déclare contre tout exclusivisme, que ce soit selon la formule de Harvey ("et de DesRochers», aurait-il pu ajouter), c'est-à-dire, exprimer en langue parfaitement française des choses toutes canadiennes, ou selon celle de Pelletier ${ }^{16}$, exprimer tout ce qu'on voit en langage canadien ${ }^{17}$. Il dit espérer un jour écrire un article pour débrouiller [...] les confusions d'idées que mes jeunes amis s'obstinent à mettre dans cette question.

15 Ce discours est reproduit dans le premier volume de Gloses critiques, Montréal, Éditions Albert Lévesque, Librairie d'action canadienne-française, 1931.

16 Dantin n'avait pas assez d'epithètes pour dire tout le mal qu'il pensait des écrits critiques d'Albert Pelletier.

17 Vers la même époque (le 12 aoùt 1931), Dantin, en réponse à des questions pośes par son ami, livrera quelques renseignements importants sur son rôle dans la publication des poèmes d'Émile Nelligan. Il annoncera même son intention d'inclure son texte sur Nelligan dans une deuxième série de Poètes d'Amérique française. Il ne le fera pas, cependant, s'arrangeant plutót, dans la deuxième série de Gloses critiques (Montréal, Éditions Albert Lévesque, Librairie d'action canadienne-française, 1935, p. 34), pour se dédire (discrètement) d'une partie de cette étude: Vous [Harry Bernard] eussiez coupé en morceaux Emile Nelligan sans lui donner l'ame d'un Chapman, et j'ai eu grand tort autrefois de lui en faire une faute. En effet, Dantin a dû être bien confus, en relisant sa préface à la suite de la dispute sur le canadianisme intégral, de retrouver sous sa propre plume des affirmations du genre: Je regrette que Nelligan n'ait pas au moins démarqué la part imitative de son auvre en donnant un cachet canadien à ses ressouvenirs étrangers, ou, plus généralement, qu'il n'ait pas pris plus près de lui ses sources habituelles d'inspiration. Sa poésie y eat gagné, certes, en personnalité et en verité. (Émile Nelligan et son cuvre, Montréal, Beauchemin, 1903 [1904], p. XIII.) 
C'est ainsi que, le 9 août 1931, DesRochers écrit une lettre où ses réflexions sur le canadianisme intégral se trouvent considérablement atténuées. On pourrait soutenir, évidemment, que c'est tout simplement par amitié pour Dantin ou par souci de clarification et que cela n'infirme en rien ses convictions profondes sur le sujet, d'autant plus qu'il propose en même temps la publication de deux volumes sur la question, où une dizaine d'écrivains québécois donneraient leurs arguments pour ou contre le canadianisme intégral (Dantin s'opposera à un tel projet). Mais comment douter qu'il ait changé d'opinion lorsqu'il affirme le 16 novembre ${ }^{18}$ :

Je sais pour ma part que j'étais en train de m'aventurer vers un "canadianisme» qui eût dégénéré en crétinisme: j’étais en train de devenir gallophobe dans les grandeurs au profit de personne et à mon détriment.

En examinant le credo que j'étais en train de me faire, je me suis aperçu qu'il était absurde. Je donnais à des détails l'importance de dogmes. Un de mes amis - que vous connaissez-est à faire un fou de lui avec son entêtement.

\section{Sur la conception de la poésie}

Par ailleurs, il s'agit d'une époque où DesRochers semble écrire peu de poésie, incertain de ce que devrait être sa voie à lui. Les lettres entre les deux amis s'espacent un peu et DesRochers ne soumet plus de poèmes au "maître" pendant plus d'un an.

On ne peut s'empêcher de se demander si le blocage dont souffre DesRochers ne découle pas en partie des différences d'opinion entre les deux amis au sujet de ce qu'est, ou devrait être, la poésie. Cette source de dissension existe de façon latente depuis le début, mais l'ascendant de Dantin sur le jeune poète tend à la masquer. Pourtant, DesRochers en semble déjà un peu conscient le 29 juillet 1929, au moment où il se déclare en extase devant tout le bien que Dantin écrit sur l'Offrande ${ }^{19}$. Ce sera plus évident dans la lettre du 15 septembre 1929 , où Dantin révèle non seulement son désaccord au sujet de Chiniquy, mais également ses réticences à l'égard de la deuxième partie d'A l'ombre de l'Orford: Je crois que votre évolution doit s'opérer principalement dans la ligne générale de votre Offrande aux

18 Déjà, le 28 septembre 1931, DesRochers disait: Je suis pratiquement forcé d'éclaircir mes idées sur le nationalisme intégral, car on m'a demandé de dire exactement ce que j'entendais par là, dans une conférence à donner au collège où j'ai commencé mes études. Je pourrais bien m'apercevoir; en fin de compte, que mon canadianisme intégral est tout simplement de la littérature tout court. Le 28 octobre, il se déclare à mi-côté de la question.

19 Ici, je pense que nous différons d'opinion. Vous voudriez me voir donner plus de liberté à mes rythmes. Moi, je veux les faire encore plus éclatants. 
vierges folles. Si le maître se montre un peu plus positif devant l'œuvre une fois imprimée, il sera surtout ravi lorsque DesRochers commence à lui envoyer les extraits d'un nouveau roman en vers qui témoignent, selon lui, d'un retour au lyrisme après le réalisme de l'Orford. Pour pouvoir commenter ce roman-poème qui, dit-il, comptera parmi les cuvres les plus fortes de DesRochers, Dantin se permet même, le 13 février 1930, de prendre une journée de congé de son travail. Et quand le jeune poète s'inquiète du caractère trop personnel de ce livre, l'aîné s'efforce de le rassurer. Sans succès, cependant ${ }^{20}$.

Néanmoins, Dantin se dédit un peu lorsque DesRochers lui envoie l'«Ode au crapaud» en juin 1930:

Je crois vraiment que c'est dans ce lyrisme épris de réalisme, à la fois objectif et chaleureux, que vous réussissez le mieux. Je préfère votre "Vent du Nord", et votre ode d'aujourd'hui, où ces deux éléments se fondent, à vos pièces purement sentimentales ou purement plastiques, et c'est là un aveu que j'ai voulu vous faire avant aujourd'hui, que j'ai même suggéré dans ma critique de vos Vierges folles. (30 juin 1930)

Entre cette citation et l'enthousiasme exprimé antérieurement en voyant DesRochers quitter le réalisme pour le lyrisme, il est intervenu un petit désaccord au sujet de Chanson citadine qui a sans doute aidé Dantin à préciser ses idées à ce sujet. DesRochers avait accueilli assez favorablement ce poème, offrant même de le multigrafier (5 mars 1930), mais avouait lui préférer Chanson javanaise. Déçu par cette réaction, Dantin cherche à se faire rassurer par d'autres amis avant de le faire publier, par l'intermédiaire de DesRochers (il lui envoie le manuscrit le 3 décembre 1930 en espétant pouvoir offrir l'œuvre imprimée à ses amis comme cadeau de Noël), mais en le dédicaçant à Robert Choquette ${ }^{21}$.

C'est lors de ce petit désaccord sur Chanson citadine que Dantin se rebiffe devant certains propos de DesRochers présentant la poésie comme une "affaire d'instinct", s'adressant à je ne saị quel sens primitif et sur laquelle il faille s'en rapporter à l'épatement des "buses» (30 juin 1930) ${ }^{22}$. Fidèle à lui-même, le maître réplique qu'il faut absolument joindre au lyrisme la pensée forte et l'expression parfaite. Il avoue d'ailleurs qu'en disant cela, il défend non seulement

20 Des fragments de ce roman-poème, "Nous avons joué dans l'ile, roman lyrique", furent publiés dans CEuvres poétiques II. Choix de poésies éparses, texte établi et annoté par Romain Legaré, Montréal, Fides, 1977, p. $111-117$ (Nénuphar).

21 On peut s'interroger sur la diplomatie d'un tel geste quand on sait que Dantin a déjà da rassurer DesRochers que sa poésie n'était pas inférieure à celle de Choquette, mais tout simplement différente (22 juin 1930).

22. Précisons que DesRochers se sert de cette définition pour expliquer pourquoi, à son avis, les buses préféreraient la poésie de Robert Choquette à la sienne. 
DesRochers contre lui-même, mais aussi Chanson citadine. Sur ce, la lettre se termine abruptement, et la correspondance ne reprendra que plus d'un mois plus tard, lorsque Dantin accepte l'offre de DesRochers d'assister à une réunion d'amis dans les cantons de l'Est. Il ajoute alors: Je sais qu'il n'y a entre nos idées qu'une très légère nuance qu'il n'est même pas important de dissiper... (5 août 1930) ${ }^{23}$

Une autre nuance fait surface en mai 1932, à l'occasion d'un commentaire où DesRochers reprochait à Choquette de ne pas faire des vers assez classiques. Dantin réplique en défendant le vers libre, source, dit-il, de chaleur et de lyrisme (15 mai 1932) ${ }^{24}$.

Mais là où Dantin aura peut-être le plus contribué à contrecarrer (ou décourager) l'évolution de DesRochers, c'est par la réaction qu'il a face à ses tentatives dans le genre "hermétique". C'est Rosaire Dion qui, à l'automne 1933, apprendra à Dantin l'existence de cette tendance chez DesRochers, car celui-ci, dont les lettres s'espacent, semble de moins en moins porté à se confesser à son aîné. Sans attendre que DesRochers en parle lui-même, Dantin écrit tout de suite pour annoncer son scepticisme devant une telle poésie. Selon lui, il s'agit d'un genre où Rimbaud, Mallarmé et Valéry ont déjà tout fait, et puis, est-ce là la poésie qui dure et mérite de durer? (19 octobre 1933) Deux mois plus tard, lorsque DesRochers lui envoie deux des poèmes hermétiques en question, accompagnés de quelques poèmes religieux, Dantin donne tout de suite sa préférence aux stances religieuses en prétextant que l'hermétisme ne puise pas aux véritables sources de la poésie, à savoir: la pensée, l'émotion, la nature, l'âme humaine (28 décembre 1933). Ces répliques rappellent d'ailleurs une autre boutade contre les gentilles pirouettes de Paul Valéry insérée par le critique dans son dithyrambe sur Walt Whitman, le 25 mai 1933.

L'allusion à Valéry rappelle que DesRochers, dans son attachement à la prosodie et dans son attirance pour une poésie plus hermétique, n'était peut-être pas si éloigné des tendances modernes de la poésie qu'on est parfois porté à le croire. Mais on ne peut récrire l'histoire et nous ne saurons jamais où l'auraient mené de telles tendances si on l'avait encouragé à les développer véritablement.

Ce qui est certain, c'est que Louis Dantin, avec son insistance sur l'importance d'une pensée claire en poésie, et Alfred DesRochers,

23 Les deux réunions à Sherbrooke auxquelles assiste Dantin semblent arriver à point pour atténuer des désaccords importants entre les deux amis.

24 Cette lettre infirme donc un peu la thèse de Jacques Blais selon laquelle le rejet de la modernité chez les poètes des années 30 proviendrait en partie de l'influence de Louis Dantin et de son opposition au vers libre ("Poètes québecois d'avant 1940 en quête de modernité ", l'Avènement de la modernité au Québec, sous la direction d'Yvan Lamonde et Esther Trépanier, Québec, Institut québécois de recherche sur la culture, 1986, p. 31). 
avec son amour des formes fixes et du sonnet, se trouvent tous les deux à côté de la voie qui, vers la même époque, portera Saint-Denys Garneau à continuer en quelque sorte le mouvement de renouvellement amorcé par Émile Nelligan et à engager la poésie québécoise sur le chemin de la "modernité".

\section{"Canadien tout court " ou Canadien français}

Il semble que 1934 ait été une année assez difficile pour DesRochers, si l'on se fie aux échos recueillis dans sa correspondance. Et c'est au beau milieu de cette année-là que son opposition aux théories présentées - par Albert Lévesque dans la Nation canadiennefrançaise le mettra en désaccord avec bon nombre de ses amis. Dans la lettre où il essaie de s'en expliquer à Dantin, il semble vraiment aux abois, se disant mal compris de tout le monde. Et je ne rime à peu près rien. (16 juillet 1934)

Lorsqu'on analyse la lettre de DesRochers en détail, son obstination à s'affirmer Canadien tout court se comprend assez facilement. Il rejette la théorie d'une nation canadienne-française comme un autre genre de racisme hitlérien et opte plutôt pour une distinction basée sur la langue. Serait Canadien (par opposition à Canadian, dit-il) tout Canadien qui parle et fonctionne en français, et non pas tout membre de la race canadienne-française quelle que soit la langue qu'il pratique. En fin de compte, DesRochers prône non pas l'assimilation des Canadiens français, comme le prétendent certains, mais l'assimilation des autres cultures au français. Une telle prise de position n'est pas sans rappeler le nationalisme prôné par Olivar Asselin et Jules Fournier vers 1915.

Dantin, qui avait déjà publié un article où il se déclarait en parfait accord avec Albert Lévesque, affirme trouver la lettre de DesRochers plutôt obscure. Il met en doute l'idée que son ami ait vraiment été mal compris et dit que, pour lui, Canadien tout court veut dire Canadien anglais, ou un Canadien français qui préfêre émigrer dans les autrés provinces. De la même façon que sur la question du canadianisme intégral, il soupçonne DesRochers de ne plus vouloir défendre les Canadiens français, d'admettre d'avance leur défaite (19 juillet 1934) ${ }^{25}$.

Il s'agit évidemment ici d'un sujet fort complexe. Il reste que Louis Dantin avait préféré rester aux États-Unis plutôt que de vivre avec les préjugés de la race canadienne-française de son époque ${ }^{26}$. Il

25 Dans la deuxième série des Gloses critiques, au chapitre sur le livre d'Albert Lévesque, Dantin présente de la même façon l'intervention du poète de Sherbrooke et lui enlève toute crédibilité en concluant qu'il devait sûrement s'agir d'une plaisanterie de la part de DesRochers.

26 En effet, il fait plutôt preuve de résistance passive lorsque DesRochers ou d'autres essaient de lui trouver un emploi qui lui permettrait de revenir au Canada. 
ne cesse de protester contre le moralisme étouffant du milieu littéraire québécois et contre le contrôle qu'y exerce le clergé ${ }^{27}$, et dit avoir tant de mal à écrire quelque chose de valable sans que cela soit considéré comme scandaleux au Québec qu'il finit par croire que son âme n'est pas une âme canadienne (1 $\mathrm{er}$ juin 1931) et par opter pour des écrits où le côté personnel se cache sous l'exotisme. Il peut donc paraître ironique que cet exilé reproche à DesRochers d'être un traître à sa race alors que celui-ci a accepté de vivre de son mieux avec les défauts de son milieu et qu'il assume sa "québécitude" de façon tout à fait réaliste et personnelle dans son œuvre. Belle illustration de la façon dont l'idéologie nationaliste dominante peut aveugler des gens très intelligents sur leurs propres illogismes idéologiques et les empêcher de comprendre chez leurs proches des écarts pourtant justifiables par rapport à cette idéologie ${ }^{28}$.

Suivront une période de quatre ans et demi de quasi-silence, puis une nouvelle série de lettres en 1939. Malgré une reprise fort prometteuse, où Dantin accueille avec grand enthousiasme les textes que DesRochers recommence à lui envoyer, cette longue correspondance se termine pour de bon le 5 décembre 1939. La dernière lettre de Dantin, quoique fort amicale, semble être restée sans réponse ${ }^{29}$. Se peut-il que DesRochers ait été offusqué quand Dantin lui demande de renvoyer tout de suite les pages manuscrites des Enfances de Fanny (que DesRochers avait même offert de faire clavigraphier) pour pouvoir les montrer à Rosaire Dion et à sa femme, Alice Lemieux ${ }^{30}$. Ou est-ce le début de la Seconde Guerre mondiale qui attira l'attention de DesRochers ailleurs? Il est difficile de le savoir de façon certaine, mais la raison principale est sûrement que cette correspondance ne jouait plus un rôle indispensable. Non seulement le bel accord des débuts se trouve-t-il fissuré par maints

27 Dantin est tellement préoccupé par cette question de la censure et du scandale, etc., qu'on commence, vers le 2 avril 1929, à croire à une obsession de sa part jusqu'au jour où, le 2 mai 1930, ces craintes se révèlent bel et bien fondées. Albert Lévesque lui-même aurait hésité, parait-il, à publier certains de ses livres, et DesRochers a dû intervenir. Quant au recueil de contes, la Vie en rêve, il sera soumis à ce boycote tacite de la part du clergé dont me parle Albert Lévesque (24 mars 1931).

28 Une contradiction semblable se trouve d'ailleurs dans la célebre préface à Émile Nelligan et son ceuvre par Louis Dantin au début du siècle où des remarques très modernistes sur la primauté de la forme côtoient des reproches. sur le manque de sujets canadiens et sur le manque de culture et de philosophie dans cette cuvre.

29 On ne peut pas en être certain, car les deux dernières lettres de DesRochers dont parle Dantin dans ses lettres à lui manquent au fonds Nadeau. En effet, la lecture des lettres de Dantin révèle qu'au moins quatre lettres de DesRochers manquent au fonds Nadeau, dont trois qui proviennent de la dernière année de leur correspondance.

30 En effet, c'est Rosaire Dion qui est devenu maintenant le légataire littéraire de Louis Dantin, et c'est lui qui assurera la publication posthume de ce roman. 
débats, mais les vies des deux hommes prennent maintenant des voies bien différentes. Dantin, mis à la retraite, se dévoue corps et âme à la littérature et, de son propre aveu, écrit plus que jamais ${ }^{31}$. DesRochers, pour sa part, se trouve de plus en plus accaparé par des besoins matériels, familiaux, et tergiverse sur le plan poétique, réticent, semble-t-il, à revenir au genre qui lui avait valu tant de renommée et d'honneurs, mais incapable de se fixer ailleurs.

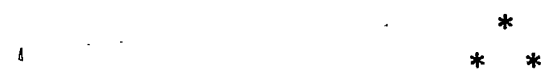

Il est indéniable que Dantin a été l'un des premiers à reconnaître le talent poétique de DesRochers. Avec la publication d'À l'ombre de l'Orford, cependant, les honneurs ne tarderont pas à venir de tous les côtés et on constate alors que, autant le rejet avait poussé DesRochers à produire et à se prouver, autant la consécration officielle semble l'avoir paralysé ${ }^{32}$.

Comment évaluer le rôle joué par Louis Dantin dans la vie d'Alfred DesRochers? En ce qui concerne la reconnaissance de l'activité littéraire et intellectuelle des cantons de l'Est, il est clair que Dantin se prêta volontiers, les premières années, au rôle que DesRochers, en tant qu'animateur culturel de cette région, voulut lui faire jouer. (Il accepte même de laisser publier sous son seul nom le texte sur «Le mouvement littéraire des cantons de l'Est » qu'il affirme plusieurs fois être autant l'œuvre de DesRochers que la sienne.) Les conséquences de ses conseils littéraires demeurent plus problématiques, d'abord parce que, quelle qu'en soit la raison, DesRochers arrête pratiquement de produire, et ensuite parce que les forces de persuasion de Louis Dantin semblent parfois aller à l'encontre des tendances naturelles de son ami.

En revanche, il est peu probable que Dantin aurait autant publié sans l'aide fort concrète que lui prêta Alfred DesRochers. Il est même permis de se demander si, sans l'encouragement de son jeune confrère, Dantin aurait connu la période de créativité littéraire qui caractérisa les dernières années de sa vie.

En réfléchissant sur la carrière littéraire d'Alfred DesRochers, il ne faut évidemment pas oublier qu'il n'est pas le seul à cette époque à

31 C'est à cette époque, lors de sa retraite prise à l'áge de 73 ans, que Dantin rédigera Fanny et 160 articles sur le "livre américain " pour le Jour, en plus de poursuivre sa collaboration à l'Avenir du Nord.

32 Voir à ce sujet l'article de Richard Giguère: «Évolution de l'horizon d'attente de la poésie du terroir: le cas de la réception critique d'À l'ombre de l'Orford d'Alfred DesRochers, 1929-1965 ", Problems of Literary Reception/Problèmes de réception littéraire, sous la direction de E. D. Blodgett et A. G. Purdy, Edmonton, Research Institute for Comparative Literature, University of Alberta, 1988, p. 102-118. 
avoir perdu en quelque sorte le feu sacré. Comme il le dira lui-même, bien plus tard, dans un petit cahier de notes:

Avec l'avènement d'Hitler, ma génération vit s'effriter tout ce en quoi elle croyait. Le silence - ou la dispersion - s'ensuivit.

La "crise» fut notre tombeau, dont seul Choquette a secoué le linceul.

La véritable explication de ce silence est sans doute beaucoup plus complexe et, au moins en partie, d'ordre littéraire. DesRochers affirmait déjà en 1931 , avec une clairvoyance étonnante, que les écrivains d'après-guerre (parmi lesquels il se classe) appartiennent à une génération de transition; ils n'ont, vis-à-vis d'œuvres antérieures, ni l'originalité de leurs devanciers ni la plénitude qu'auront leurs successeurs (Paragraphes, p. 173). Il souligne cependant, avec raison, l'importance de ce rôle de transition.

Trop modernistes pour leur époque et pas assez pour celle d'aujourd'hui, Alfred DesRochers et Louis Dantin auront quand même grandement contribué, chacun à sa façon, à faire évoluer l'institution littéraire québécoise. 